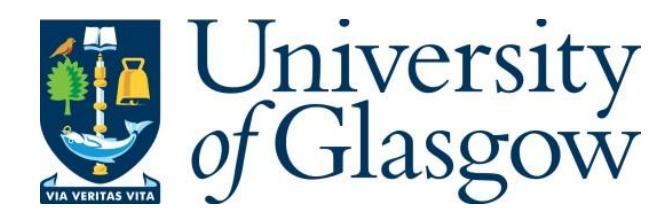

Wood, T. A., Hesse, H. and Smith, R. S. (2017) Predictive control of autonomous kites in tow test experiments. IEEE Control Systems Letters, 1(1), pp. 110-115.

There may be differences between this version and the published version. You are advised to consult the publisher's version if you wish to cite from it.

http://eprints.gla.ac.uk/150468/

Deposited on: 21 November 2017

Enlighten - Research publications by members of the University of Glasgow

http://eprints.gla.ac.uk 


\title{
Predictive Control of Autonomous Kites in Tow Test Experiments
}

\author{
Tony A. Wood ${ }^{1}$, Henrik Hesse ${ }^{2}$, and Roy S. Smith ${ }^{1}$
}

\begin{abstract}
In this paper we present a model-based control approach for autonomous flight of kites for wind power generation. Predictive models are considered to compensate for delay in the kite dynamics. We apply Model Predictive Control (MPC), with the objective of guiding the kite to follow a figureof-eight trajectory, in the outer loop of a two level control cascade. The tracking capabilities of the inner-loop controller depend on the operating conditions and are assessed via a frequency domain robustness analysis. We take the limitations of the inner tracking controller into account by encoding them as optimisation constraints in the outer MPC. The method is validated on a kite system in tow test experiments.
\end{abstract}

\section{INTRODUCTION}

Airborne Wind Energy (AWE) generators have been proposed as a mobile, cost-effective, and more sustainable alternative to conventional wind turbines. In this work we focus on kite power systems which generate power by flying a multi-line tethered wing or kite in crosswind motion following a figure-of-eight pattern. The tethers are connected to winches on the ground which generate power by unreeling the lines in the so-called traction phase. The traction phase is alternated with a retraction phase to reel in the tethers using only a fraction of the power generated during traction, leading to a net positive cycle power.

Since the original inception of the kite power concept [1], several groups have developed prototype systems and the reference book [2] provides a detailed overview of the field of AWE. In this work we focus on the control of kites during the traction phase of a ground-based system, as developed in [3], with ground-based measurements of line angles and length, steering actuation, and power generation in the socalled ground station (GS). The experimental implementation of autonomous kite power systems requires stabilising control approaches which can handle the unstable, nonlinear dynamics inherent in kite systems. Model-free guidance approaches based on a switching point strategy and using the kite heading angle as feedback variable for tracking [4], [5] provide a successful starting point for further control development.

The performance of such model-free approaches, however, is affected by varying operating conditions and time delay which make tuning difficult and effectively limit the overall system power output, especially for ground-based systems.

\footnotetext{
*This research was supported by the Swiss National Science Foundation (Synergia) No. 141836.

${ }^{1}$ T. A. Wood and R. S. Smith are with the Automatic Control Laboratory (IfA), ETH Zurich, Physikstrasse 3, 8092 Zurich, Switzerland. E-mail: \{woodt, rsmith\}@control.ee.ethz.ch. Tel: +41 446323629.

${ }^{2} \mathrm{H}$. Hesse is with the Aerospace Sciences Division, University of Glasgow (Singapore), 510 Dover Road, Singapore 139660 (formerly at IfA, ETH Zurich). Email: henrik.hesse@glasgow.ac.uk
}

To improve the control performance in experimental implementations of kite power prototypes, model-based control approaches have been proposed in [5]-[8]. The underlying kinematic models link the kite heading angle to the overall kite motion. In [6] the estimated kite heading angle was further related to the steering input with a model that includes an input delay. State estimation for ground-based AWE system with output delay is addressed in [9].

In [6]-[8], [10], [11] guidance strategies have been developed for experimental kite power systems to allow path following. In particular, [7] used a kinematic model including input delay for a figure-of-eight path planning and tracking strategy. To ensure robust performance of the control approach we further considered limitations on the tracking bandwidth imposed by the input delay. The robustness of the cascaded control architecture of [6], [7] was further improved following a Model Predictive Control (MPC) approach in [11] by formulating the path following problem as an optimisation problem with constraints on the heading angle, its rate of change, and the kite position.

MPC is naturally suited to address the complexities in the control of kites, e.g. constraint satisfaction and minimisation of a tracking error, and has been extensively explored in simulation in [12]-[15]. The application of MPC approaches to kite power systems, however, tends to be sensitive to unmodelled dynamics and hindered by limitations in processing power for real-time operation. In [11] we therefore use the kinematic model introduced in [7] which allows for online adaptation of model parameters to reduce the model mismatch. The approach was demonstrated in simulation to achieve successful path following while satisfying constraints imposed by the limitations of a lower-level tracking controller that are subject to model uncertainty and input delay.

The contribution of this paper is the extension of the kinematic model to account for variations of the kite velocity within a figure-of-eight cycle which significantly improves the tracking capabilities of the path following controller developed in [11]. Moreover, we demonstrate the performance of the MPC approach for varying line length during tow test experiments with a prototype kite power system. To our knowledge this is the first experimental implementation of an MPC scheme on an AWE system.

The remainder of the paper is structured as follows. In Section II we describe the cascaded control architecture with delay compensation and constrained outer-loop guidance that accounts for limitations of the inner-loop controller. In Section III we describe the implementation of the control scheme for tow test experiments. We finally present experimental results in Section IV before concluding in Section V. 


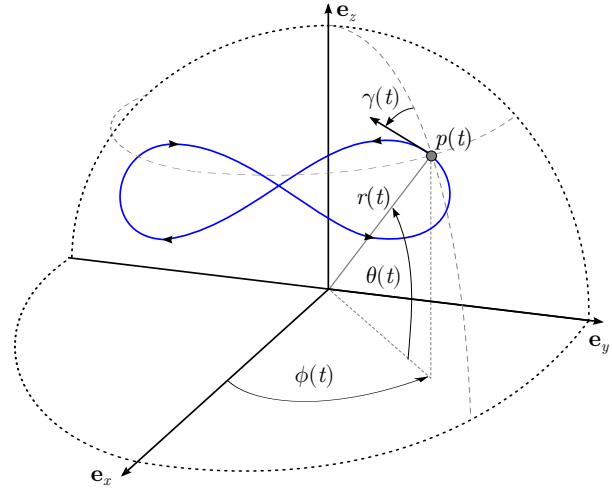

Fig. 1: Kite position, $p(t)$, and heading angle, $\gamma(t)$.

\section{Control Scheme}

We consider a two line kite power system in the traction phase where the kite is flown in crosswind conditions. The motion of the kite perpendicular to the tethers is actuated by the difference in lengths of the two lines, $\delta$. The position of the kite in Cartesian coordinates, $p$, can be expressed as a function of the elevation angle, $\theta$, the azimuth angle, $\phi$, and the line length, $r$, which are illustrated in Figure 1 and all measured from the GS in practice,

$$
p(t)=\left[\begin{array}{c}
p_{x}(t) \\
p_{y}(t) \\
p_{z}(t)
\end{array}\right]=\left[\begin{array}{c}
r(t) \cos (\theta(t)) \cos (\phi(t)) \\
r(t) \cos (\theta(t) a) \sin (\phi(t)) \\
r(t) \sin (\theta(t))
\end{array}\right] .
$$

A cascaded architecture is considered to control the flight trajectory of the kite around a figure-of-eight path. Controlling the heading angle, also referred to as velocity vector orientation, defined as

$$
\gamma:=\arctan \left(\frac{\cos (\theta) \dot{\phi}}{\dot{\theta}}\right),
$$

has been shown to be an effective approach for autonomous crosswind flight control of kites [4]-[6]. The outer loop of the cascade is controlled by the guidance controller which produces a commanded heading angle trajectory, $\gamma^{\mathrm{cmd}}(t)$, that is tracked in the inner loop by the tracking controller. Figure 2 illustrates the control architecture.

The steering behaviour of the kite is affected by line dynamics due to the indirect actuation via tethers. This effect can be modelled as an input delay and taken into account in a model-based delay compensation scheme [6]. The presence of delay and model uncertainty, however, imposes fundamental limitations on the tracking performance. As in [11] we assess the limitations of the tracking controller, based on estimates of the current operating conditions, with a frequency domain robustness analysis. We parametrise the limitations by an upper bound on the rate of change of the commanded signal that can be tracked. The resulting rate limit, $l_{r}$, is communicated to the optimisation-based guidance controller which takes the current inner-loop tracking capabilities into account as a constraint in the generation of the commanded heading angle trajectory.

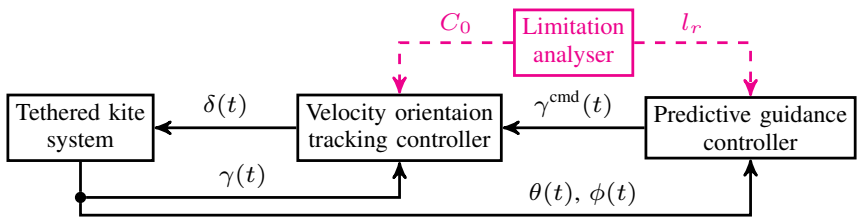

Fig. 2: Cascaded control architecture with inner-loop controller tracking a commanded heading angle, $\gamma^{\mathrm{cmd}}(t)$, given by the outer-loop guidance controller which incorporates the limitations of an inner loop as constraint, $\left|\dot{\gamma}^{\mathrm{cmd}}(t)\right|<l_{r}$.

\section{A. Control Models}

The evolution of the kite position expressed in terms of line angles, $\xi(t):=(\theta(t), \phi(t))$, can be modelled by the following kinematic unicycle model, [7],

$$
\begin{aligned}
\dot{\theta}(t) & =\frac{v_{\theta \phi}(t)}{r} \cos (\gamma(t)), \\
\dot{\phi}(t) & =\frac{v_{\theta \phi}(t)}{r \cos (\theta(t))} \sin (\gamma(t)),
\end{aligned}
$$

where the velocity perpendicular to the tethers is modelled as a static function of the position, $\xi(t)$, and orientation, $\gamma(t)$,

$$
v_{\theta \phi}(t)=r \alpha_{L} \cos (\theta(t)) \cos (\phi(t))-r \alpha_{G} \cos (\gamma(t)),
$$

with parameters $\alpha_{L}, \alpha_{G}>0$ representing velocity components arising from lift and gravitational forces. Modelling the velocity to vary over a cycle captures the true behaviour more closely than the assumption of constant velocity made in the control model of [7], [11] as can be seen in the experimental results shown in Figure 6a.

Based on experimental observations in [6], we relate the heading angle, $\gamma(t)$ to the steering input, $\delta(t)$, by integrator dynamics with a time delay $t_{d}$,

$$
\dot{\gamma}(t)=K \delta\left(t-t_{d}\right) .
$$

The model parameters, $r, \alpha_{L}, \alpha_{G}, K$, and $t_{d}$ are assumed to change only slowly such that they can be considered constant within the control horizon and are re-identified online based on updated measurements.

\section{B. Tracking Controller}

To control of the heading angle we account for the delay in (4) in a model-based approach as described in [6]. We predict the orientation after the delay time, $\gamma^{t_{d}}(t)$, and apply a proportional gain controller to the difference of the commanded and the predicted output,

$$
\delta(t)=C_{0}\left(\gamma^{\mathrm{cmd}}(t)-\gamma^{t_{d}}(t)\right) .
$$

This approach of controlling delayed systems based on model predictions is referred to as predictor feedback [16]. To assess the performance of the tracking controller we consider the the regulation of the time-shifted tracking error, $e_{t_{d}}(t):=$ $\gamma^{\mathrm{cmd}}(t)-\gamma\left(t+t_{d}\right)$.

The control model in (4) is simple but there is a considerable degree of uncertainty in the steering gain parameter, $K$, and delay, $t_{d}$, which limits the tracking performance. We 
therefore model the uncertainty of the plant, $\gamma=G(s) \delta$, with

$$
G(s)=G_{0}(s) e^{-s t_{d}}=\frac{K}{s} e^{-s t_{d}},
$$

in the frequency domain to be a weighted multiplicative perturbation with the perturbation weight for delayed firstorder systems introduced in [17],

$$
\begin{aligned}
\mathcal{G} & :=\left\{\left(1+W_{m}(s) \Delta(s)\right) G(s) \mid\|\Delta(s)\|_{\infty} \leq 1\right\}, \\
W_{m}(j \omega) & = \begin{cases}\left|\frac{K+\delta K}{K} e^{-j \delta t_{d} \omega}-1\right| & \text { if } \omega<\frac{\pi}{\delta t_{d}}, \\
\left|\frac{K+\delta K}{K}\right|+1 & \text { if } \omega \geq \frac{\pi}{\delta t_{d}},\end{cases}
\end{aligned}
$$

where $\delta K, \delta t_{d}$ are the bounds on the deviations of the model parameters, and $\Delta(s)$ is the unknown but bounded perturbation.

The prediction feedback in (5) corresponds to a Smith predictor [18] and can be written in the frequency domain as, $\delta=C(s)\left(\gamma^{\text {cmd }}-\gamma\right)$, with

$$
C(s)=\frac{C_{0}}{1+C_{0} G_{0}(s)\left(1-e^{-s t_{d}}\right)} .
$$

For all perturbed plants, $G^{p}(s) \in \mathcal{G}$, the closed-loop relationship between the input and the shifted tracking error is, $e_{t_{d}}=S_{t_{d}}^{p}\left(s \mid C_{0}\right) \delta$, with

$$
S_{t_{d}}^{p}\left(s \mid C_{0}\right)=\frac{1+L(s)\left(1-e^{s t_{d}}\right)+L(s)\left(1-e^{s t_{d}}\right) W_{m}(s) \Delta(s)}{1+L(s)+L(s) W_{m}(s) \Delta(s)},
$$

where $L(s)=C(s) G(s)$ is the loop transfer function.

The tracking capabilities depend on the control gain, $C_{0}$, and on the properties of the commanded signal, $\gamma^{\mathrm{cmd}}(t)$. We would like to set the control gain and constrain the commanded signal such that $\left|e_{t_{d}}(t)\right|<l_{e}$ for all perturbed plants in the set given in (6). In particular, we limit the magnitudes of the commanded signal, $\left|\gamma^{\mathrm{cmd}}(t)\right|<l_{m}$, and its rate of change, $\left|\dot{\gamma}^{\mathrm{cmd}}(t)\right|<l_{r}$. These limits on the timedomain signals are approximately translated to the frequency domain with the robust performance condition

$$
\sup _{\|\Delta\|_{\infty} \leq 1}\left\|W_{p}\left(j \omega \mid l_{r}\right) S_{t_{d}}^{p}\left(j \omega \mid C_{0}\right)\right\|_{\infty}<1,
$$

where we define the performance weight to be

$$
W_{p}\left(j \omega \mid l_{r}\right)= \begin{cases}\frac{l_{m}}{b_{e}} & \text { if } \omega<\frac{l_{r}}{l_{m}}, \\ \frac{l_{r}}{b_{e} \omega} & \text { if } \omega \geq \frac{l_{r}}{l_{m}} .\end{cases}
$$

Upper and lower bounds for the the left-hand side of (7) can be determined by a structured singular value analysis. Given values of the model parameters, $K, t_{d}$, the level of uncertainty considered, $\delta K, \delta t_{d}$, and the bounds $l_{m}, l_{e}$, the condition in (7) depends on the nominal control gain, $C_{0}$, and the rate limit, $l_{r}$, which are to be adjusted such that robust tracking performance is guaranteed, $\left(C_{0}, l_{r}\right) \in \mathcal{R} \mathcal{P}$, with

$$
\mathcal{R P}:=\left\{(\zeta, \rho) \in \mathbb{R}^{2} \mid \sup _{\|\Delta\|_{\infty} \leq 1}\left\|W_{p}(j \omega \mid \rho) S_{t_{d}}^{p}(j \omega \mid \zeta)\right\|_{\infty}<1\right\} .
$$

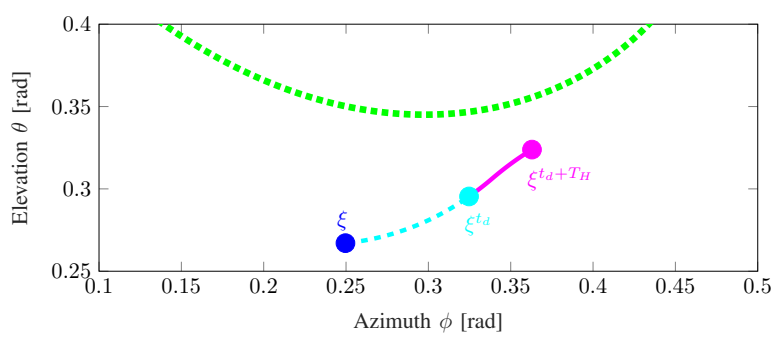

Fig. 3: Prediction of flight trajectory: delay compensation (dashed), MPC prediction (solid); reference path (dotted).

\section{Predictive Guidance Control}

For the design of the guidance control we assume that the tracking controller is able to follow the commanded signal well but delayed by the steering delay, i.e., $\gamma(t) \approx$ $\gamma^{\mathrm{cmd}}\left(t-t_{d}\right)$. We apply the model given in (2) and (3) to compensate for the delayed tracking. Given a prediction of the kite position and orientation $t_{d}$ ahead of time, $\xi^{t_{d}}(t)=$ $\left(\theta^{t_{d}}(t), \phi^{t_{d}}(t)\right)$ and $\gamma^{t_{d}}(t)=\gamma^{\mathrm{cmd}}(t)$ respectively, we find a value for the commanded signal at the current time, $\gamma^{\mathrm{cmd}}(t)$, that controls the kite to follow a reference figure-of-eight path, $\xi^{\text {ref }}=\left(\phi^{\text {ref }}, \theta^{\text {ref }}\right)$. The reference path is generated and updated online such that the corresponding reference orientation, $\gamma^{\text {ref }}$, is of sinusoidal form and its rate of change satisfies the limitation given by the tracking controller as suggested in [7]. This implies that for lower limits on the rate of change, larger figure-of-eight paths are required.

We apply a MPC approach with the objective of minimising the deviation of the delay compensating prediction, $\xi^{t_{d}}(t)$, from the reference path, $\xi^{\text {ref }}$, over a finite time horizon, $T_{H}$, while constraining the kite to remain in a predefined safety window, $\underline{\xi} \leq \xi^{t_{d}}(t) \leq \bar{\xi}$, and satisfying the limit on the rate of change of the commanded heading angle imposed by the tracking controller, $\left|\dot{\gamma}^{\mathrm{cmd}}(t)\right|<l_{r}$. We also constrain the magnitude of the commanded orientation, $\left|\gamma^{\mathrm{cmd}}(t)\right|<l_{m}$, to avoid commanding the kite to fly straight down towards the ground. More details on the optimisation problem that is formulated and solved to capture the guidance objective are presented in Section III-B. Figure 3 illustrates the guidance concept with the predicted path which the kite is expected to follow. The prediction is split into two parts: the first part is determined by the past inputs in the delay compensation scheme; the second part consists of the further evolution due to current and future actuation and can be optimised by the MPC.

\section{EXPERIMENTAL IMPLEMENTATION}

MPC for autonomous kites has been studied extensively in literature [12]-[14]. Demonstration of the considered controllers is however limited to simulation. In this work we therefore design and conduct experiments to validate the applicability of predictive crosswind flight control approaches to experimental kite power prototypes. The experimental 

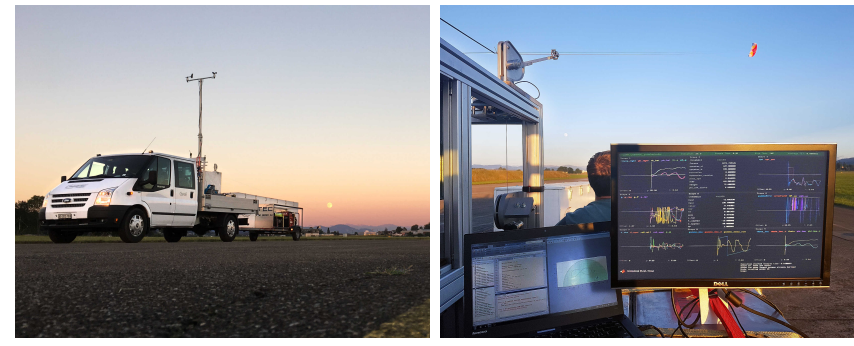

Fig. 4: Tow test configuration with kite connected to GS control system (right) mounted on a moving vehicle (left).

implementation further demonstrates the real-time capability of the proposed MPC as described in Section III-B. We further demonstrate a novel experimental method to test kite controllers in still wind conditions using the tow test configuration as described next.

\section{A. Tow Test Experiments}

The experimental flight control tests were performed on a prototype AWE system [3] which has been developed at Fachhochschule Nordwestschweiz (FHNW) and modified to allow for tow test experiments as depicted in Figure 4. By mounting the GS on the back of a truck we can create relative wind by moving the vehicle. The origin of the coordinate frame shown in Figure 1 moves with the truck and the $x$ axis points in the opposite direction of the vehicle velocity. For the results presented in this work, a HQ Apex III $5 \mathrm{~m}^{2}$ ram air kite was connected to the GS via two tethers with a maximum length of $150 \mathrm{~m}$.

The forces on the tethers are controlled by regulating the reel-out speed of the winches. The winch control is independent of the steering control. The entire control and estimation architecture required for the steering controller is implemented in Matlab Simulink and runs on a Speedgoat Real-Time Target Machine with a fixed sampling time, $T=$ 0.01 s. With this mobile two-line configuration we can test in wind still conditions and create reproducible wind scenarios to develop controller for crosswind flight of kites with variable tether length.

\section{B. Controller Implementation}

As part of the predictive control approach in this work, we use real-time measurement data to identify and update the control model parameters online in an adaptive fashion. Given estimates of the model parameters, the tracking control gain, $C_{0}$, and the rate limit on the commanded heading angle, $l_{r}$, are set such that robust tracking performance is guaranteed. We use a sufficient analytic condition derived in [11] to determine suitable values for $\left(C_{0}, l_{r}\right) \in \mathcal{R} \mathcal{P}$. The condition involves an upper bound on the left-hand side of (7) which is explicitly parametrised by the tuning variables $C_{0}$ and $l_{r}$, allowing for simple evaluation online.

The kite dynamics are discretised with the forward $\mathrm{Eu}-$ ler method, $\xi_{k+1}=f_{d}\left(\xi_{k}, \gamma_{k}\right)$. The figure-of-eight reference path is parametrised by a periodic sequence of positions, $\xi^{\text {ref }}=\left(\xi_{1}^{\text {ref }}, \xi_{2}^{\text {ref }}, \ldots, \xi_{N}^{\text {ref }}\right)$, and orientations, $\gamma^{\text {ref }}=$ $\left(\gamma_{1}^{\text {ref }}, \gamma_{2}^{\text {ref }}, \ldots, \gamma_{N}^{\text {ref }}\right)$, that satisfy the model dynamics, $\xi_{i+1}^{\text {ref }}=$ $f_{d}\left(\xi_{i}^{\mathrm{ref}}, \gamma_{i}^{\mathrm{ref}}\right)$, for $i=0,1, \ldots, N-1$, and $\xi_{1}^{\mathrm{ref}}=f_{d}\left(\xi_{N}^{\mathrm{ref}}, \gamma_{N}^{\mathrm{ref}}\right)$.

To capture the guidance objective, we consider a system describing the deviation of the prediction of the kite position after the delay from the reference path with state $\chi_{k}:=\xi_{k}^{t_{d}}-\xi_{j}^{\text {ref }}$ and input $u_{k}:=\gamma_{k}^{\text {cmd }}-\gamma_{j}^{\text {ref }}$, where $\left(\xi_{j}^{\text {ref }}, \gamma_{j}^{\text {ref }}\right)$ represent the reference point closest to the prediction of the kite state after the delay time, $j:=$ $\arg \min _{q \in\{1,2, \ldots, N\}} \operatorname{dist}\left(\left(\xi_{k}^{t_{d}}, \gamma_{k}^{\mathrm{cmd}}\right),\left(\xi_{q}^{\text {ref }}, \gamma_{q}^{\text {ref }}\right)\right)$. Minimising the deviation of the position prediction from the reference path corresponds to controlling the deviation system to zero.

We linearise the kite kinematics around the reference path, starting at the reference point closest to the prediction of the kite position after the delay time, to obtain the linear time varying system $\chi_{i+1} \approx A_{i} \chi_{i}+B_{i} u_{i}$, with $A_{i}:=\frac{\partial f_{d}}{\partial \xi}\left(\xi_{j+i}^{\mathrm{ref}}, \gamma_{j+i}^{\text {ref }}\right)$ and $B_{i}:=\frac{\partial f_{d}}{\partial \gamma}\left(\xi_{j+i}^{\text {ref }}, \gamma_{j+i}^{\text {ref }}\right)$, for $i=0,1, \ldots, H-1$, where $H$ is number of discrete time steps in the prediction horizon. To encode the input rate constraint into the optimisation problem we augment the deviation state such that the difference in inputs is an input to the augmented system, $\hat{\chi}_{i+1}=\hat{A}_{i} \hat{\chi}_{i}+\hat{B}_{i} \Delta u_{i}$, with $\Delta u_{i}:=u_{i}-u_{i-1}$,

$$
\hat{\chi}_{i}:=\left[\begin{array}{c}
\chi_{i} \\
u_{i-1}
\end{array}\right], \quad \hat{A}_{i}:=\left[\begin{array}{cc}
A_{i} & B_{i} \\
0 & 1
\end{array}\right], \quad \hat{B}_{i}:=\left[\begin{array}{c}
B_{i} \\
1
\end{array}\right] .
$$

The following optimisation captures the guidance task,

$$
\begin{array}{cl}
\min _{\Delta u, \epsilon} & \sum_{i=0}^{H-1}\left(\hat{\chi}_{i}^{\top} \hat{Q} \hat{\chi}_{i}+\epsilon_{i}^{\top} S \epsilon_{i}\right)+\hat{\chi}_{H}^{\top} \hat{Q}_{H} \hat{\chi}_{H}+\epsilon_{H}^{\top} S_{H} \epsilon_{H}, \\
\text { s.t. } & \hat{\chi}_{i}=\hat{A}_{i} \hat{\chi}_{i}+\hat{B}_{i} \Delta u_{i}, \\
& \underline{\chi}_{i} \leq \chi_{i}+\epsilon_{i}, \quad \chi_{i}-\epsilon_{i} \leq \bar{\chi}_{i}, \quad \epsilon_{i} \geq 0, \\
\underline{u}_{i} & \leq u_{i} \leq \bar{u}_{i}, \\
\Delta u_{i} & \leq \Delta u_{i} \leq \overline{\Delta u}_{i},
\end{array}
$$

with slew rate sequence $\Delta u=\left(\Delta u_{0}, \Delta u_{1}, \ldots, \Delta_{H-1}\right)$, slack variables sequence $\epsilon=\left(\epsilon_{0}, \epsilon_{1}, \ldots, \epsilon_{H}\right)$, state limits $\underline{\chi}_{i}:=\underline{\xi}-\xi_{j+i}^{\mathrm{ref}}, \bar{\chi}_{i}:=\bar{\xi}-\xi_{j+i}^{\mathrm{ref}}$, input limits $\underline{u}_{i}:=-l_{m}-\gamma_{j+i}^{\mathrm{ref}}$, $\bar{u}_{i}:=\bar{l}_{m}-\gamma_{j+i}^{\text {ref }}$, slew rate limits $\Delta u_{i}:=-l_{r} T-\gamma_{j+i}^{\text {ref }}+$ $\gamma_{j+i-1}^{\text {ref }}, \overline{\Delta u}_{i}:=l_{r} T-\gamma_{j+i}^{\text {ref }}+\gamma_{j+i-1}^{\text {ref }}$, and positive-definite weighting matrices $S, S_{H}, \hat{Q}:=\operatorname{diag}(Q, R), \hat{Q}_{H}:=$ $\operatorname{diag}\left(Q_{H}, R\right)$, where $\left(Q, Q_{H}\right)$ penalise the position deviation, $R$, penalises the orientation deviation, and $\left(S, S_{H}\right)$ penalise the slack variables.

The inclusion of the slack variables into the optimisation problem makes the position constraints soft. The state constraints can be violated with high penalisation $S \gg$ $Q, S_{H} \gg Q_{H}$ which prevents the constraint optimisation problem from becoming infeasible in situations where there is no possibility of keeping the kite in the desired position window. This is relevant when initialising the controller from arbitrary positions. Note that the slew rate is constrained but not explicitly penalised in the cost function. The optimisation problem (8) is implemented and solved online with the optimisation software generation tool FORCES Pro [19].

The value of the current commanded heading angle at time step $k$ is determined by the first element of the sequence $\Delta u^{*}$ that obtains the minimum of $(8), \gamma_{k}^{\mathrm{cmd}}=\Delta u_{0}^{*}+\gamma_{k-1}^{\mathrm{cmd}}-\gamma_{j-1}^{\text {ref }}+$ 


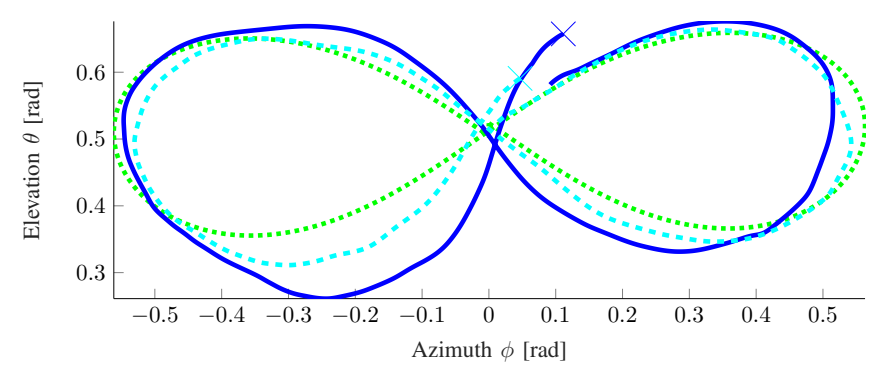

Fig. 5: Trajectory tracking results (Flight 1): kite position trajectory, $\xi(t)$ (solid), following a reference figure-of-eight path, $\xi^{\text {ref }}$ (dotted), with delay compensation prediction trajectory, $\xi^{t_{d}}(t)$ (dashed). The trajectories start at the position marked with crosses at time $t=0 \mathrm{~s}$.

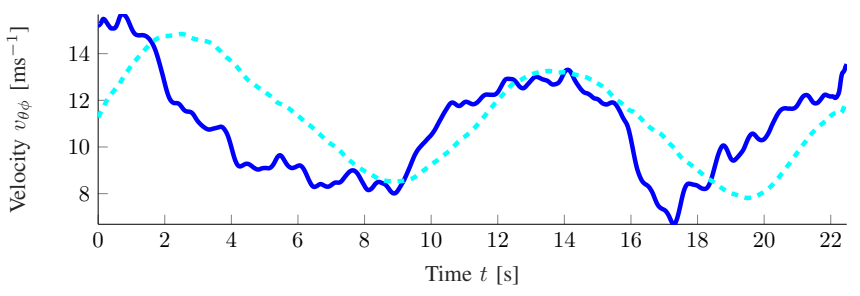

(a) Velocity trajectory, $v_{\theta \phi}(t)$, estimated based on derivative of position measurements, $r(t) \sqrt{\dot{\theta}^{2}(t)+\cos (\theta(t)) \dot{\phi}^{2}(t)}$ (solid), and based on model (3) (dashed).

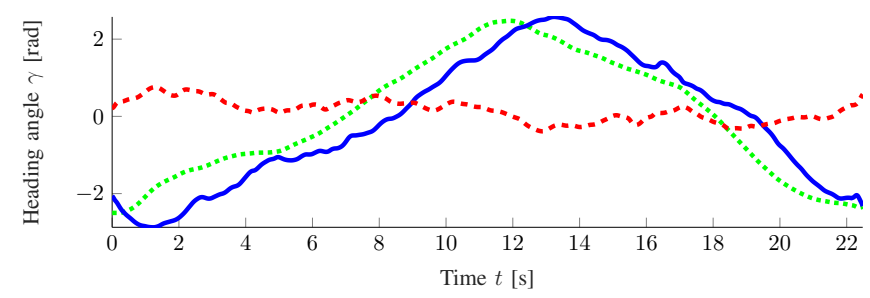

(b) Trajectory of heading angle, $\gamma(t)$ (solid), tracking commanded signal, $\gamma^{\mathrm{cmd}}(t)$ (dotted), with shifted tracking error, $e_{t_{d}}(t)$ (dashed).

Fig. 6: Trajectory of velocity and heading angle (Flight 1 ).

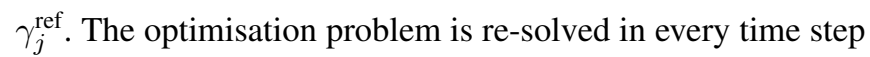
with a receding horizon.

\section{Results}

The predictive control scheme has been experimentally tested in tow test experiments. In this section we present the results of an experiment conducted on the runway of St. Stephan airport in Switzerland on 9 December 2016. Data from two test flights (Flight 1 and Flight 2) are presented.

The number of stages in optimisation problem (8) was selected to be $H=30$, resulting in a MPC prediction horizon of $T_{H}=0.3 \mathrm{~s}$. Note that the overall prediction horizon of the guidance controller consists of the sum of the delay compensation and the MPC horizon; with an average estimated delay of approximately $t_{d}=0.7 \mathrm{~s}$ the overall prediction horizon stretches over approximately $1 \mathrm{~s}$. The weights of the MPC objective were $Q=\operatorname{diag}(1,2), Q_{H}=$ $5 \cdot Q, R=5 \cdot 10^{-3}, S=10^{5} \cdot Q, S_{H}=10^{5} \cdot Q_{H}$. The safety window was defined by the position bounds $\underline{\theta}=0.17 \mathrm{rad}$, $\bar{\theta}=1.40 \mathrm{rad}, \underline{\phi}=-0.70 \mathrm{rad}$, and $\bar{\phi}=0.70 \mathrm{rad}$. The limit on the commanded heading angle was set to be $l_{m}=2.5 \mathrm{rad}$ and the desired maximum tracking error was selected to be $l_{e}=0.9 \mathrm{rad}$. The model parameters were updated online two times per figure-of-eight cycle with least-squares fits to realtime measurements. The uncertainty levels of the parameters of the steering model were set to be $20 \%$ of the estimated parameter values, $\delta K=0.2 \cdot K, \delta t_{d}=0.2 \cdot t_{d}$.

Figure 5 shows the tracking of the reference figure-ofeight path over one cycle (Flight 1). We observe that the kite position trajectory, $\xi(t)$, follows the reference path, $\xi^{\text {ref }}$. The delay compensated prediction of the position, $\xi^{t_{d}}(t)$, follows the reference path more closely as it is the signal used in the MPC objective. The difference between the trajectories of the position and its prediction can be explained by model mismatch and to a greater extend by the tracking error in the inner-loop controller. For the same flight interval (Flight 1 ), the velocity perpendicular to the tethers, $v_{\theta \phi}(t)$, obtained from the derivative of the line angle measurements, and its prediction based on (3) are shown in Figure 6a. The significant velocity variation is captured by the velocity model in (3) which leads to better predictions compared to the assumption of constant velocity. The tracking of the commanded heading angle during the cycle (Flight 1) is illustrated in Figure $6 \mathrm{~b}$. The heading angle, $\gamma(t)$ follows the commanded reference well with a time shift.

Considering a longer flight duration (Flight 2), we can observe the adaptation to changing operating conditions. Figure 7 shows a flight trajectory over 3 minutes. As the wind speed and line length change, the parameter estimates vary, shown in Figure 8a, and the bound on the rate of change of the commanded orientation, shown in Figure 8b, is adapted according to the changing limitations of the tracking controller. We observe that the controller is able to track figure-of-eights cycles while the wind speed changes between $2.7 \mathrm{~m} / \mathrm{s}$ and $6.6 \mathrm{~m} / \mathrm{s}$ to demonstrate adaptation to varying wind conditions. Throughout the experiment the line length is reeled out from $79 \mathrm{~m}$ to $100 \mathrm{~m}$. For larger line lengths the estimate of the delay increases in general. For fast increases of the wind speed, however, the tether forces increase resulting in less line sag and a lower delay estimate despite the line reeling out. In this case the limit on the rate of the commanded signal decreases, as evident for $80-100 \mathrm{~s}$ in Figure 8, resulting in a slower turn rate and larger flight path. The results indicate that good tracking performance can be achieved by constraining the guidance based on the limitation of the tracking controller and by adapting reevaluating this for changing operating conditions.

\section{CONCLUSion}

We have presented a model-based control approach to fly kites autonomously in crosswind conditions for power generation. The control problem is split into two parts with a cascaded control architecture. We apply predictor feedback to account for the input delay affecting the system. To ensure that the commanded signal determined by the outer control loop is not too fast for the inner control loop to track, the 


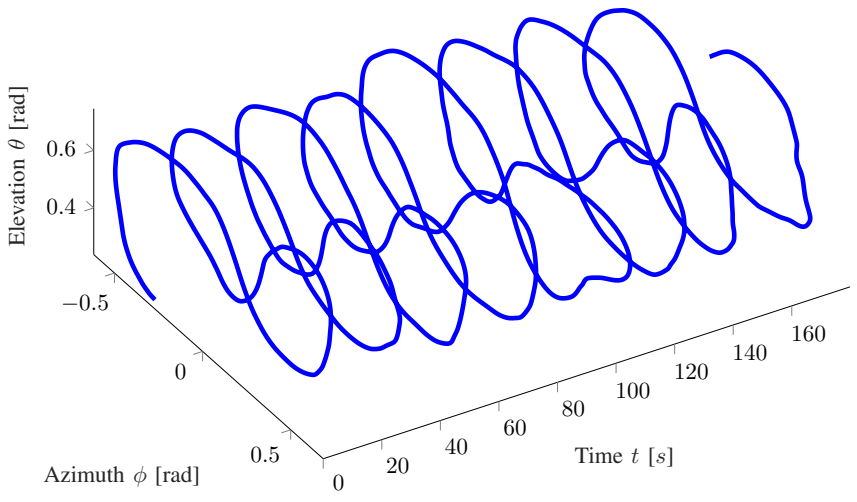

Fig. 7: Long duration position trajectory, $\xi(t)$ (Flight 2).

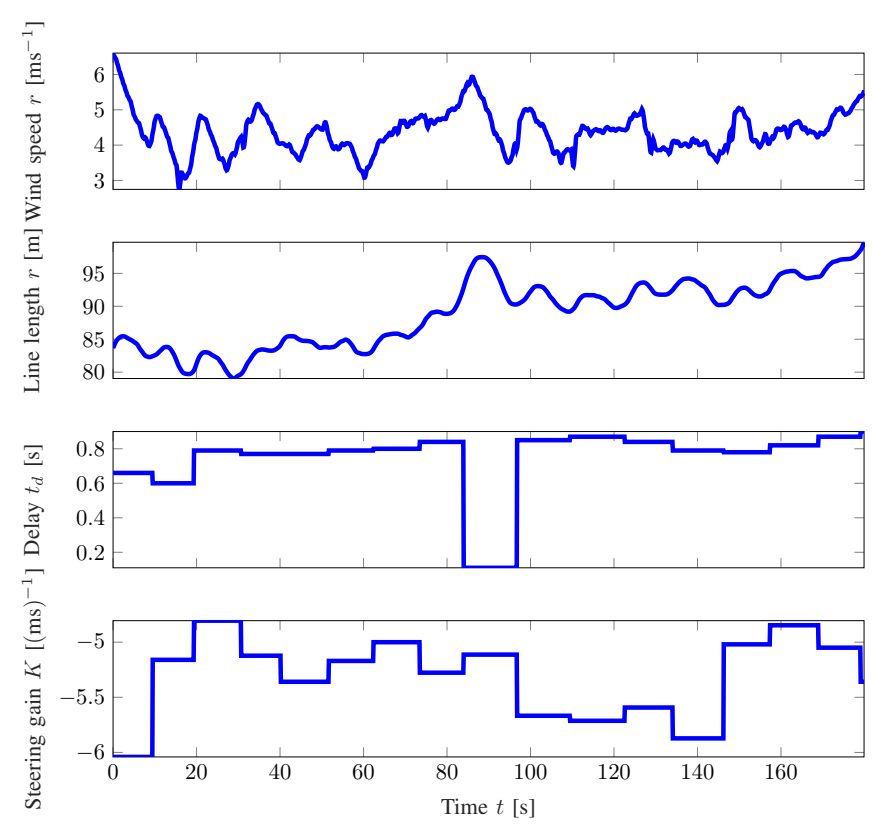

(a) Measurements of the wind speed, $w$, and the line length, $r$; estimates of the parameters in the steering model (4).

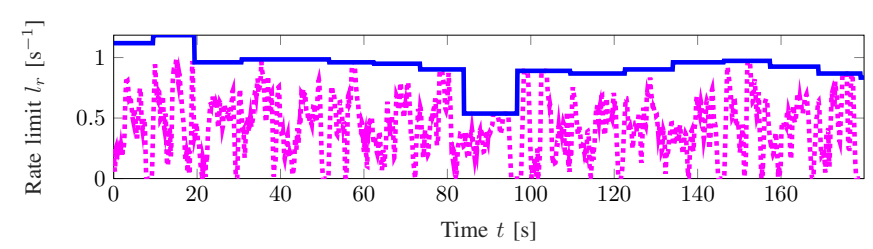

(b) Bound on the rate of change of the commanded heading angle, $l_{r}$ (solid), and resulting magnitude of the rate of the commanded orientation, $\left|\dot{\gamma}^{\mathrm{cmd}}(t)\right|$ (dotted).

Fig. 8: Evolution of parameters (Flight 2): operating conditions and parameter estimates (8a), resulting limit on the rate of change of the commanded signal (8b).

limitations of the inner-loop controller are determined using a robustness analysis based on model parameter uncertainty. The limitations are parametrised as a bound on the maximum rate of change of the commanded signal. This bound is taken into account as a constraint in the model predictive outer-loop guidance control.

The main benefit of applying model predictive control in this approach is constraint satisfaction. The optimisation framework, however, also enables the consideration of new objectives. The approach has been successfully tested in tow test flight experiments. The optimisation-based guidance strategy is able to steer the kite to follow figure-of-eight paths. By adapting model parameters and control constraints online using updated measurements, autonomous flights under strongly varying operating conditions were achieved.

\section{ACKNOWLEDGEMENTS}

The authors would to thank J. Heilmann, D. Aregger, I. Lymperopoulos, and S. Diwale (A2WE project [3]) for their contribution in conducting the flight experiments. Support from embotech $\mathrm{GmbH}$ and in particular A. Hempel is gratefully acknowledged.

\section{REFERENCES}

[1] M. L. Loyd, "Crosswind Kite Power," Journal of Energy, vol. 4, 1980

[2] U. Ahrens, M. Diehl, and R. Schmehl, Airborne Wind Energy. Springer, 2013.

[3] "Autonomous Airborne Wind Energy Project (A2WE)," http://a2we.skpwiki.ch, accessed 31. October 2015.

[4] L. Fagiano, A. U. Zgraggen, M. Morari, and M. Khammash, "Automatic Crosswind Flight of Tethered Wings for Airborne Wind Energy: Modeling, Control Design, and Experimental Results," IEEE Transactions on Control Systems Technology, vol. 22, no. 4, pp. 14331447, 2014.

[5] M. Erhard and H. Strauch, "Flight control of tethered kites in autonomous pumping cycles for airborne wind energy," Control Engineering Practice, vol. 40, 2015.

[6] T. A. Wood, H. Hesse, A. U. Zgraggen, and R. S. Smith, in American Control Conference, Chicago, IL, USA.

[7] — in Conference on Decision and Control, Osaka, Japan, Dec.

[8] N. Rontsis, S. Costello, I. Lymperopoulos, and C. N. Jones, in Conference on Decision and Control, Osaka, Japan, Dec.

[9] M. Polzin, T. A. Wood, H. Hesse, and R. S. Smith, in IFAC World Congress, Toulouse, France, July 2017. [Online]. Available: https://polybox.ethz.ch/index.php/s/rwgFNsWDAfFsHjm

[10] S. Costello, G. Francois, and D. Bonvin, "Directional Real-Time Optimization Applied to a Kite-Control Simulation Benchmark," in European Control Conference, Linz, Austria, July 2015.

[11] T. A. Wood, E. Ahbe, H. Hesse, and R. S. Smith, "Predictive Guidance Control for Autonomous Kites with Input Delay," in IFAC World Congress, Toulouse, France, July 2017. [Online]. Available: https://polybox.ethz.ch/index.php/s/RES8RafsbZO7q2L

[12] M. Diehl, H. G. Bock, and J. P. Schlöder, "A real-time iteration scheme for nonlinear optimization in optimal feedback control," SIAM Journal on Control and Optimization, vol. 43, no. 5, pp. 1714-1736, 2005.

[13] M. Canale, L. Fagiano, and M. Milanese, "High altitude wind energy generation using controlled power kites," IEEE Transactions on Control Systems Technology, vol. 18, no. 2, pp. 279-293, 2010.

[14] A. Ilzhöfer, B. Houska, and M. Diehl, "Nonlinear MPC of kites under varying wind conditions for a new class of large-scale wind power generators," International Journal of Robust and Nonlinear Control, vol. 17, no. 17, pp. 1590-1599, 2007.

[15] M. Zanon, S. Gros, and M. Diehl, "Model Predictive Control of RigidAirfoil Airborne Wind Energy Systems," in Airborne Wind Energy. Springer, 2013.

[16] M. Krstic, Delay compensation for nonlinear, adaptive, and PDE systems. Springer, 2009.

[17] D. L. Laughlin, D. E. Rivera, and M. Morari, "Smith predictor design for robust performance," International Journal of Control, vol. 46, no. 2, pp. 477-504, 1987.

[18] O. J. Smith, "A Controller to Overcome Dead Time," ISA Journal, vol. 6, pp. 28-33, 1959.

[19] A. Domahidi and J. Jerez, "FORCES Professional," embotech GmbH (http://embotech.com/FORCES-Pro), July 2014. 